\title{
NÍVEIS DE GASTOS E EFICIÊNCIA PÚBLICA EM EDUCAÇÃO: UM ESTUDO DE MUNICÍPIOS PAULISTAS UTILIZANDO ANÁLISE ENVOLTÓRIA DE DADOS
}

Data de submissão: 10/10/2018 Aceite: 01/11/2018

Alberto Borges Matias ${ }^{1}$ Gislaine de Miranda Quaglio²

Bruno Garcia de Oliveira ${ }^{3}$ João Paulo Resende de Lima ${ }^{4}$

Renan Vilela Bertolin ${ }^{5}$

\section{RESUMO}

A qualidade da educação ofertada pelo setor público é importante, pois tem impacto na formação do cidadão e na geração de capital humano. Neste âmbito, estudar o nível de gasto público e a eficiência da gestão permite identificar melhores práticas, além de reforçar a avaliação do setor público como mecanismo de controle social e efetividade do Estado. O presente artigo tem o objetivo de avaliar o nível de gasto em educação municipal em relação à eficiência na promoção de Educação Básica de qualidade. Foram analisados 47 municípios paulistas, através da Análise Envoltória de Dados (DEA) num modelo utilizando três inputs e seis outputs para os anos de 2007, 2009 e 2011. Os resultados indicam aumento do gasto total em educação, porém, a maior alocação de recursos não proporcionou melhores resultados, tendo em vista a aplicação ineficiente.

Palavras-chave: Educação, Eficiência Pública, Análise Envoltória de Dados.

\footnotetext{
1 Possui graduação em Administração de Empresas pela Universidade de São Paulo - USP, mestrado em Administração pela Universidade de São Paulo e doutorado em Administração de Empresas pela Universidade de São Paulo. Professor titular da Faculdade de Economia, Administração e Contabilidade da Universidade de São Paulo. Ribeirão Preto-SP.Brasil. E-mail: matias@usp.br

2 Possui graduação em Ciências Econômicas pelo Centro Universitário Moura Lacerda, graduação em Gestão Financeira pelo Centro Universitário Moura Lacerda, mestrado em Economia pela Universidade Estadual Paulista Júlio de Mesquita Filho - UNESP/FCLAR. Doutoranda em Economia Aplicada pela Faculdade de Economia, Administração e Contabilidade da Universidade de São Paulo(USP-RP), Ribeirão Preto-SP. Brasil. E-mail: gislaine.dqm@gmail.com 3 Possui graduação em Administração pela Universidade Federal de Goiás, UFG, mestrado em Administração de Organizações pela Faculdade de Economia Administração e Contabilidade de Ribeirão Preto/USP. Doutorando na Faculdade de Economia Administração e Contabilidade de Ribeirão Preto/USP. Professor da Universidade Federal de Goiás - Centro de Gestão e Negócios CGEN/UFG-RC). E-mail:brunogarcia@fearp.usp.br

4 Possui graduação em Ciências Contábeis pela Faculdade de Economia, Administração e Contabilidade de Ribeirão Preto, FEARP/USP, mestrado em Controladoria e Contabilidade pela FEA-RP/USP. Integrante do Núcleo FEA de Pesquisa em GÊNEro, RAça e Sexualidade (GENERA) e Núcleo de Pesquisas e Estudos em Controle Gerencial (NUPECON - ESAN/UFMS). Doutorando no Programa de Pós-Graduação em Controladoria e Contabilidade pela FEA/USP na linha de "Ensino e Pesquisa em Contabilidade". São Paulo-SP. E-mail: jp.resendelima@hotmail.com

5 Possui graduação em Licenciatura em Química pela Universidade Federal de São Carlos, UFSCar. Foi Professor de Educação Básica da Secretaria de Educação do Estado de São Paulo, SEE/SP. São Carlos-SP. Brasil. E-mail:renanvile@hotmail.com
} 


\section{INTRODUÇÃO}

A avaliação do setor público é um tema importante, pois demonstra onde e como são aplicadas as rendas obtidas através dos tributos recolhidos. Para Ramos e Schabbach (2012), ao avaliar o setor público tem-se como interesse a melhoria da eficiência do gasto público e, por meio do controle social, busca-se a efetividade da ação do Estado e a melhoria da qualidade de gestão.

No setor educativo o tema é de suma importância em razão da influência do setor público na formação do cidadão e do capital humano. O investimento de recursos e tempo na educação escolar busca garantir que os indivíduos colham os seus benefícios por meio de empregos melhor remunerados, satisfação no trabalho ou pelo fato de adquirir um conhecimento maior do mundo que o circunda (BONAMINO et al., 2010). Nessa perspectiva, diversos pesquisadores têm se dedicado a avaliar e compreender a relação entre o nível de gastos em educação e o desempenho escolar (MARLOW, 2000; UNNEVER; KERCKHOFF; ROBINSON, 2000; KIM, 2001; WOESSMANN, 2003), além disso, torna-se importante avaliar a eficiência na aplicação destes recursos.

É no contexto da importância da educação, do nível de gastos da gestão pública e da avaliação da eficiência da gestão pública que o artigo se sustenta. Tem-se como objetivo avaliar o nível de gastos em educação municipal em relação à eficiência na promoção de uma Educação Básica de qualidade, por meio dos recursos da Análise Envoltória de Dados (DEA), tal como realizado por Sampaio e Guimarães (2009), Rosano-Penã et al. (2012) e Wilbert e D’Abreu (2013).

$\mathrm{O}$ artigo está estruturado em quatro tópicos principais. Na primeira parte, apresenta-se uma revisão da literatura, para compreensão de variáveis importantes no desempenho escolar, e estudos sobre a eficiência pública na promoção da educação, assim como pesquisas que buscaram mensurar a eficiência da gestão pública na geração de resultados educacionais satisfatórios. A segunda apresenta o método, a coleta e a análise de dados. A terceira discute os resultados e, por fim, são apresentadas as conclusões.

\section{REVISÃO DA LITERATURA}

A revisão de literatura está dividida em duas partes principais. A primeira aborda o desempenho escolar, com ênfase na importância do nível de gasto público em educação e seus impactos na qualidade educacional. A segunda parte discute eficiência pública em educação e apresenta estudos anteriores que buscaram mensurar a eficiência da gestão pública na geração de resultados educacionais.

\section{FATORES QUE INFLUENCIAM NO DESEMPENHO ES- COLAR}

A conversão de investimento financeiro (capital econômico) em capital humano por meio da educação tem sido estudada por diversos pesquisadores. Em 1966, o Relatório Coleman, um estudo publicado nos Estados Unidos, rompeu com o dogma de que haveria uma relação significativa entre o desempenho dos alunos e os investimentos em educação, assim como provocou uma "ruptura com a visão otimista de construção de uma sociedade igualitária pela via da educação para todos" (BONAMINO et al., 2010, p. 487). Desde a publicação do Relatório Coleman, pesquisadores em diversos países têm se dedicado ao tema, na busca por confirmar ou refutar as informações.

Segundo Kim (2001), os estudos posteriores ao Relatório Coleman ignoram dois fatos: a) o método utilizado (na maioria dos estudos, análise por regressão) para medir a eficácia dos 
recursos escolares é inadequado e b) maiores recursos na educação podem fazer com que pais reduzam o tempo e/ou investimentos na educação de seus filhos. $O$ autor observou que, quando ocorre um aumento nos recursos educacionais, as mães de maior escolaridade não mudam seu tempo com as crianças, mas as mães com menor escolaridade diminuem o seu tempo de cuidado, nas palavras do pesquisador, "os recursos educacionais não têm só um efeito direto sobre o desempenho dos alunos, mas também um efeito indireto sobre o desempenho dos alunos através de seu poder de alterar a dinâmica familiar" (ibid., p. 80).

Unnever, Kerckhoff e Robinson (2000) desenvolveram um estudo analisando os dados de distritos escolares por meio de regressão, com base em procedimentos de mínimos quadrados ponderados, no que diz respeito ao status socioeconômico da população e sua influência no desempenho dos alunos. Diante dos resultados, os autores propuseram um modelo que considera três fatores que influenciam no desempenho escolar: as características socioeconômicas, as habilidades conceituais prévias e os recursos destinados à educação.

Woessmann (2003) analisou um conjunto de dados de mais de 250.000 estudantes em 39 países e concluiu que a qualidade da educação é influenciada por uma série de fatores: presença de exames centralizados; distribuição do poder de decisão entre escolas e órgãos governamentais; influência dos professores e sindicatos na política educacional; distribuição do poder de decisão sobre o sistema de educação entre níveis de governo e grau de competição entre escolas públicas e privadas. $O$ autor ainda sugere que a questão crucial não é fornecer mais recursos, mas melhorar o ambiente institucional em que as escolas funcionam, e ressalta que "um sistema educacional em que todas as pessoas têm um incentivo para melhorar o desempenho do estudante é a única alternativa que promete efeitos positivos" (ibid., p. 74).

No Brasil, Barros et al. (2001) realizaram um estudo com jovens das regiões Nordeste e Sudeste, para investigar o impacto de quatro fatores no desempenho educacional: a qualidade e disponibilidade dos serviços, o custo de oportunidade do tempo, a disponibilidade de recursos familiares e a disponibilidade de recursos da comunidade. Segundo os autores, as comunidades com melhores condições financeiras afetam positivamente o desempenho escolar de seus membros, pois há adultos com escolaridade maior que auxiliam nas tarefas escolares. Dentre os resultados, podemos destacar que a escolaridade dos pais é fator impactante no desempenho dos jovens em questão, visto que "o impacto sobre o desempenho educacional de um ano a mais de escolaridade dos pais supera o impacto de três anos a mais de escolaridade dos professores" (ibid., p. 35). No que diz respeito aos professores, o estudo revela que a escolaridade dos professores exerce influência, sendo mais marcante no Ensino Fundamental do que no Ensino Médio. Assim, deve-se reforçar a qualidade dos serviços educacionais de base na formação escolar dos alunos.

Já Albernaz, Ferreira e Franco (2002), por meio da aplicação de modelos hierárquicos lineares a uma base de dados do Sistema de Avaliação da Educação Básica (SAEB), buscaram compreender os fatores que impactam no desempenho dos alunos. Os dados foram agrupados em dois grupos: papel das características individuais e familiares e o papel das variáveis escolares e dos professores. No que diz respeito ao primeiro grupo, o nível socioeconômico é determinante no desempenho dos alunos, não apenas o nível da família, mas também o nível socioeconômico da clientela da escola, que quanto mais alto, maior é o desempenho médio dos alunos. Em relação às variáveis escolares, tanto a qualidade do professor (nível de escolaridade) quanto a qualidade da estrutura física contribuem para um melhor desempenho dos alunos. 


\section{EFICIÊNCIA PÚBLICA EM EDUCAÇÃO}

Por contribuir na formação de capital humano e social, torna-se pertinente avaliar periodicamente os sistemas educativos existentes para adequá-los às novas necessidades da sociedade. Além da avaliação periódica, atualmente se exige que o sistema educativo produza cada vez mais e que a qualidade desta produção seja sempre melhorada (DELORS et al., 1998).

Para mensurar a produção do sistema educativo é possível aplicar uma medida de eficiência que relaciona o quanto é produzido com o quanto poderia ser produzido num nível de máxima produtividade, utilizando assim os insumos da melhor maneira possível (RIBEIRO FILHO, et al., 2010). Analisar a produção educacional é notoriamente difícil por dois motivos: a educação envolve a produção de diversos outputs e há certa dificuldade em mensurá-los. Nos métodos de estimativa da eficiência escolar destaca-se ainda que os conhecimentos a respeito de quais fatores influenciam os alunos são inadequados ou de difícil mensuração (BIFULCO; BRETSCHNEIDER, 2001).

Waldo (2007) argumenta que a eficiência técnica estima a produtividade máxima e, desta forma, a eficiência pode ser considerada a distância entre a produção de uma unidade produtora e de uma unidade com melhor aproveitamento. Conroy e Arguea (2008) corroboram com o pensamento de Waldo (2007) e complementam que a principal diferença entre a eficiência técnica e a eficiência econômica é o fato de a primeira desconsiderar os preços dos insumos e produtos.

Zoghbi et al. (2009) estudaram o desempenho dos Estados no tocante aos gastos públicos com educação por meio do conceito de eficiência relativa, aplicando indicadores do SAEB, como distorção idade-série, taxa de concluintes e taxa de atendimento para mensurar a eficiência de cada estado e comparar os escores obtidos. Os autores concluíram que os Estados com melhores desempenhos acadêmicos nem sempre são os mais eficientes em relação aos gastos, contudo, este resultado sugere que há a possibilidade de melhorar a eficiência caso sejam adotadas melhores técnicas de gestão. Os resultados se alinham com os encontrados por Silva, Souza e Araújo (2013), que analisaram 26 capitais brasileiras para verificar se as mais eficientes tinham melhor desempenho no ensino fundamental e concluíram que não existe relação entre estas variáveis.

Neste contexto de mensuração de eficiência, têm crescido a utilização da Análise Envoltória de Dados (DEA). A técnica foi inicialmente desenvolvida por Farrell (1957), contudo, ganhou notoriedade após o estudo de Charnes, Cooper e Rhodes (1978). A DEA é uma técnica não paramétrica que define uma fronteira de eficiência a partir de múltiplos inputs e outputs para cada uma de suas unidades tomadoras de decisão (Decision Making Unit - DMU). Segundo Saurin et al. (2013), esta metodologia foi inicialmente aplicada em instituições públicas, devido à dificuldade de alocação de preços a insumos e produtos, impossibilitando assim a mensuração da eficiência. Estudos utilizam o DEA como modelo em amplas áreas da sociedade, de setores comerciais a comparações entre municípios (DIDONET; VILLAVICENCIO, 2008, VILLAVICENCIO, DIDONET 2008).

No modelo de DEA, conhecido como CCR, assume-se que os rendimentos da escala são constantes, ou seja, a variação nos inputs causa uma variação de mesma proporção nos outputs (CHARNES; COOPER; RHODES, 1978). Já Banker, Charnes e Cooper (1984) propuseram um modelo conhecido como BCC, aplicável aos casos em que os rendimentos de escala são variáveis e não considera a proporcionalidade entre inputs e outputs. A eficiência obtida pela DEA é relativa e baseia-se em observações reais do conjunto de DMUs. As DMUs que melhor utilizam seus recursos formam a fronteira de eficiência enquanto as demais têm sua ineficiência calculada com base na sua distância da fronteira. Segundo Faria, Jannuzzi e Silva (2008, p. 158) "a DEA permite que se calcule a eficiência de cada DMU, ao realizar comparações entre as unidades do grupo analisado, no intuito de destacar as melhores dentro dele". 
Especificamente em Educação, Sampaio e Guimarães (2009) empregaram a metodologia da DEA para comparar a eficiência de escolas públicas e privadas na região metropolitana de Recife, capital de Pernambuco. Os autores adotaram uma amostra de 23.040 alunos que prestaram o vestibular para ingressar na Universidade Federal de Pernambuco (UFPE), sendo 76,58\% alunos de escolas privadas e $23,42 \%$ de escolas públicas. A partir da decomposição do índice de eficiência, os pesquisadores concluíram que existe uma grande distinção entre escolas públicas e privadas de ensino, já que as escolas privadas obtiveram eficiência máxima e as públicas ficaram a uma distância de $10 \%$ da fronteira de eficiência, explicitando a necessidade de melhorias no ensino público. Os autores ainda ressaltam que os institutos federais obtiveram rendimento melhor que os colégios estaduais (SAMPAIO; GUIMARÃES, 2009).

Rosano-Peña, Albuquerque e Marcio (2012) estudaram a eficiência dos gastos em educação através da comparação de resultados obtidos por meio da metodologia DEA e do método da fronteira invertida. Os autores adotam como período de análise os anos entre 2005 a 2009 e sua amostra são 246 redes municipais de ensino nos municípios goianos. Os resultados apontam uma ineficiência global de $67,44 \%$ e que se as escolas tivessem adotado melhores práticas de gestão alcançariam os mesmos resultados, contudo, economizando R\$ 178 milhões. Concluíram que a maior alocação de recursos não proporciona melhores resultados se estes forem aplicados de forma ineficiente.

Wilbert e D'Abreu (2013) aplicaram a metodologia DEA para mensurar a eficiência dos gastos públicos na educação de 57 municípios alagoanos. Os autores concluíram que os municípios com baixo Produto Interno Bruto (PIB) per capita e baixo valor gasto por aluno foram os mais eficientes no estudo, reforçando a ideia de que o montante do investimento não impacta na qualidade da educação.

Blackburn, Brennan e Ruggiero (2014) realizaram um estudo para estimar a eficiência de escolas primárias e secundárias na Austrália. O modelo, utilizando Análise Envoltória de Dados, foi construído com um fator condicional em que escolas localizadas em ambientes com melhores indicadores socioeconômicos não eram utilizadas como benchmarking para escolas em ambientes piores. Os autores sugerem que este fator busca reduzir o viés de comparação de eficiência em função de variáveis não controláveis. Os resultados indicaram que escolas australianas são moderadamente ineficientes e que a eficiência aumenta para os $20 \%$ de escolas com o ambiente mais favorável. Além disso, observa-se que ganhos de eficiência ocorrem na medida em que se aumenta o número de matrículas. Em média escolas primárias gastam cerca de $20 \%$ acima do custo mínimo. Nestas condições os autores identificaram uma economia potencial de 1.903 dólares por aluno nas escolas analisadas (BLACKBURN; BRENNAN; RUGGIERO, 2014).

Harrison e Rouse (2014) por sua vez realizaram uma análise de eficiência de escolas da Nova Zelândia utilizando como inputs os recursos escolares e como outputs o desempenho dos alunos. Adicionalmente, o modelo de comparação também foi estratificado em função das características socioeconômicas dos estudantes. A pesquisa constatou que o desempenho médio escolar tende a ser maior quando as escolas estão localizadas em áreas em que as escolas competem de maneira acirrada por alunos com os melhores desempenho acadêmico, tanto entre escolas públicas quanto com escolas privadas. Para os autores, estes resultados contrastaram com outras pesquisas internacionais ao encontrar evidências de que a competição leva a melhores desempenhos. No entanto, estes resultados aparentemente variaram em função do tamanho da escola, sugerindo que a competição pode aumentar a lacuna entre as escolas com melhores e piores desempenhos. A longo prazo, isso pode resultar em aumento da ineficiência de recursos públicos fixos, já que a sobrevivência das escolas públicas é indiretamente relacionada ao desempenho (HARRISON; ROUSE, 2014). 


\section{METODOLOGIA: ANÁLISE ENVOLTÓRIA DE DADOS}

Este estudo tem como objetivo verificar a eficiência dos municípios paulistas na aplicação de recursos na área de educação nos anos 2007, 2009 e 2011. As datas avaliadas foram definidas em função das três edições do Índice de Desenvolvimento da Educação Básica (IDEB). O IDEB é um indicador de qualidade da educação que combina informações a respeito da aprovação em cada período com os resultados obtidos em uma prova padronizada (Prova Brasil) aplicada às séries finais de cada ciclo. A aplicação da prova ocorre bianualmente, com avaliação para alunos da 4a e 8a série de escolas que possuem o mínimo de 20 alunos matriculados (INEP, 2014).

Optou-se pela Análise Envoltória de Dados (DEA) como método por relacionar múltiplos inputs e outputs na análise de eficiência de uma Unidade Tomadora de Decisões (DMU). Faz-se necessário definir as Unidades Tomadoras de Decisão (DMU), os inputs e os outputs empregados, assim como o tipo e a orientação do modelo para a compreensão desse estudo.

\subsection{Unidades Tomadoras de Decisão (DMU)}

Em virtude das políticas de descentralização ocorridas pós-Constituição de 1988, a gestão pública municipal passou a ser a principal responsável pelas políticas sociais voltadas à educação, saúde e assistência social (RIBEIRO, 2009). Segundo os dados divulgados pelo Instituto Brasileiro de Geografia e Estatística (IBGE) em 2014, o estado de São Paulo conta com 645 municípios, desta forma foi considerado a priori a população total de municípios como DMU. Nem todos os municípios, entretanto, disponibilizam dados completos para os três anos propostos, restando, assim, uma amostra de 48 municípios. Por incongruência nos dados de inputs coletados (os valores divulgados pelo município e os dados enviados divergiam), o município de São Paulo foi desconsiderado, restando 47 DMUs para análise.

\subsection{Definição de Inputs e Outputs}

Os inputs, Quadro 1, foram definidos com base no trabalho de Rosano-Peña, Albuquerque e Marcio (2012), que teceram uma análise similar da qualidade da educação para municípios do Estado de Goiás. Busca-se, com estes indicadores, abranger despesas com manutenção e funcionamento das escolas, pagamento de funcionários e professores, além do dispêndio de recursos voltado à aquisição de bens duráveis.

\begin{tabular}{|c|c|}
\hline INPUT & $\begin{array}{c}\text { Despesa de Pessoal } \\
\text { Outras Despesas Correntes } \\
\text { Investimentos de Capital }\end{array}$ \\
\hline & Taxa de Aprovação nos anos iniciais \\
TUTPUT & Taxa de Aprovação nos anos finais \\
& Média padronizada da Prova Brasil nos anos iniciais \\
& Média padronizada da Prova Brasil nos anos finais \\
& Número de matrículas realizadas nos anos iniciais \\
Número de matrículas realizadas nos anos finais
\end{tabular}

Quadro 1. Inputs e Outputs utilizados na análise.

Fonte: Adaptado de Rosano-Peña, Albuquerque e Marcio (2012).

Por sua vez, os outputs utilizados se relacionam com a quantidade de resultado produzido (medido pelo número de matrículas e taxa de aprovação), bem como a qualidade da educação (medida pelo resultado da prova padronizada). Foram considerados, para cada um dos indicado- 
res, os resultados nos anos iniciais e finais. Os outputs são os mesmos utilizados no trabalho de Rosano-Peña, Albuquerque e Marcio (2012).

\subsection{Tipo e orientação do modelo}

Para mensurar a eficiência das DMUs foi aplicado o modelo de retorno variável de escala (BBC ou VRS), que torna possível mensurar a ineficiência técnica associada à habilidade gerencial (AMBROZINI; NAGANO; MERLO, 2003), e o modelo com retorno constante de escala (CCR), com intuito de avaliar as ineficiências em escala relativa ao porte das instituições. Na avaliação da ineficiência de escala também foi utilizado o teste Wilcoxon de diferença de mediana para amostras independentes.

Quanto à orientação, foram utilizados modelos orientados para inputs e também para outputs. A orientação para input possibilita identificar as DMUs que mantém um melhor nível de resultado com um consumo menor de recursos (WILBERT; D’ABREU, 2013). Já a orientação para output, estima os melhores resultados possíveis com o nível de gasto atual.

\section{COLETA E ANÁLISE DE DADOS}

Os dados utilizados no estudo foram coletados conforme descrito:

- Os dados dos municípios, como nome e população, foram coletados no IBGE (2014);

- A coleta dos dados referente aos gastos municipais (inputs) foi realizada por meio do site do Sistema de Informações sobre Orçamentos Públicos em Educação - SIOPE (FNDE, 2014). Como os dados não estavam disponíveis, as informações foram solicitadas ao Fundo Nacional de Desenvolvimento da Educação pelo site usando como prerrogativa a lei de transparência de dados públicos 12.527/11 (BRASIL, 2011).

- As médias normalizadas da Prova Brasil e a Taxa de Aprovação (IDEB) foram obtidas através de informações divulgadas pelo Instituto Nacional de Estudos e Pesquisas Educacionais Anísio Teixeira (INEP, 2014).

- O número de matrículas realizadas na 4a e 8a séries do Ensino Fundamental de cada município foi coletado no site da Fundação Sistema Estadual de Análise de Dados SEADE (SEADE, 2014).

Em relação à análise de dados, as informações foram agrupadas numa base de dados única e analisados por meio de gráficos e tabelas. Na fase de estatística descritiva, foi utilizado o programa estatístico "Software Action" versão 2.4.163.322, que opera em plataforma R (PORTAL ACTION, 2012). Para a resolução dos modelos de DEA, utilizou-se diretamente a plataforma $R$ com auxílio do package "Benchmarking", desenvolvido por Bogetoft e Otto (2011).

\section{ANẤLISE E DISCUSSÃO}

\subsection{Inputs e Outputs}

Os inputs utilizados referem-se às despesas de pessoal, investimento e outras despesas correntes. Em média, $60 \%$ dos valores são destinados para despesas de pessoal, ademais, observa-se que, para cada um real em investimento, gasta-se sete com despesas de pessoal. Ressalta-se, contudo, que as faixas de gasto mínimo percentual costumam ser definidas pela Lei 
de Responsabilidade Fiscal. Além do gasto entre as três contas analisadas, observa-se na Figura 1 o aumento do valor total despendido em educação entre 2007 e 2011. Para o grupo analisado, em 2007 o gasto total era de 1,25 bilhões e em 2011 chegou a 1,69 bilhões, um crescimento de $31 \%$ (valores reais, atualizados para junho/2014).

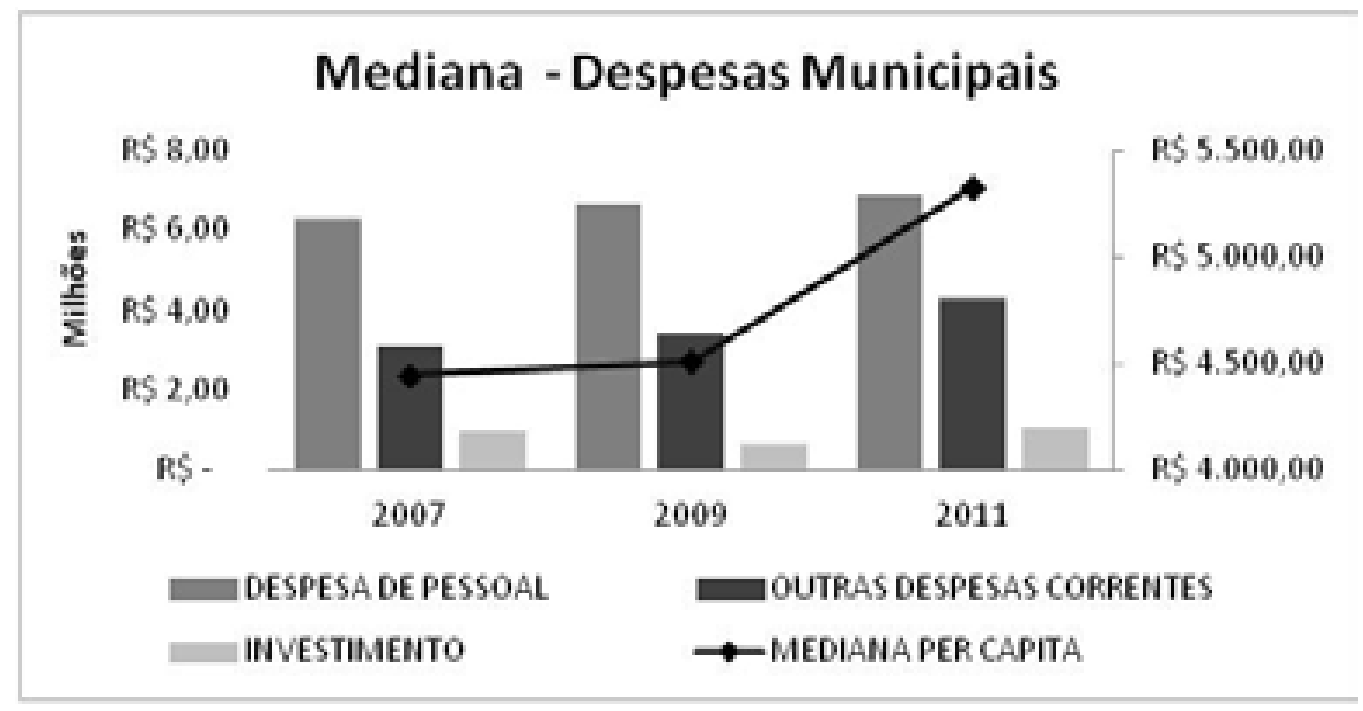

Figura 1. Despesas Municipais (2007-2011)

Fonte: Elaborado pelos autores com dados do FNDE (2014)

Com relação ao dispêndio per capita, a Figura 2 aponta o avanço da mediana e da média entre 2007 e 2011. A mediana per capita, calculada a partir do número de alunos matriculados, avançou de $R \$$ $4.447,39$ em 2007 para $\mathrm{R} \$ 5.326,22 \mathrm{em}$ 2011. Desta forma, o aumento de gastos observado na Figura 1 não ocorreu proporcionalmente ao aumento do número de alunos matriculados no mesmo período.

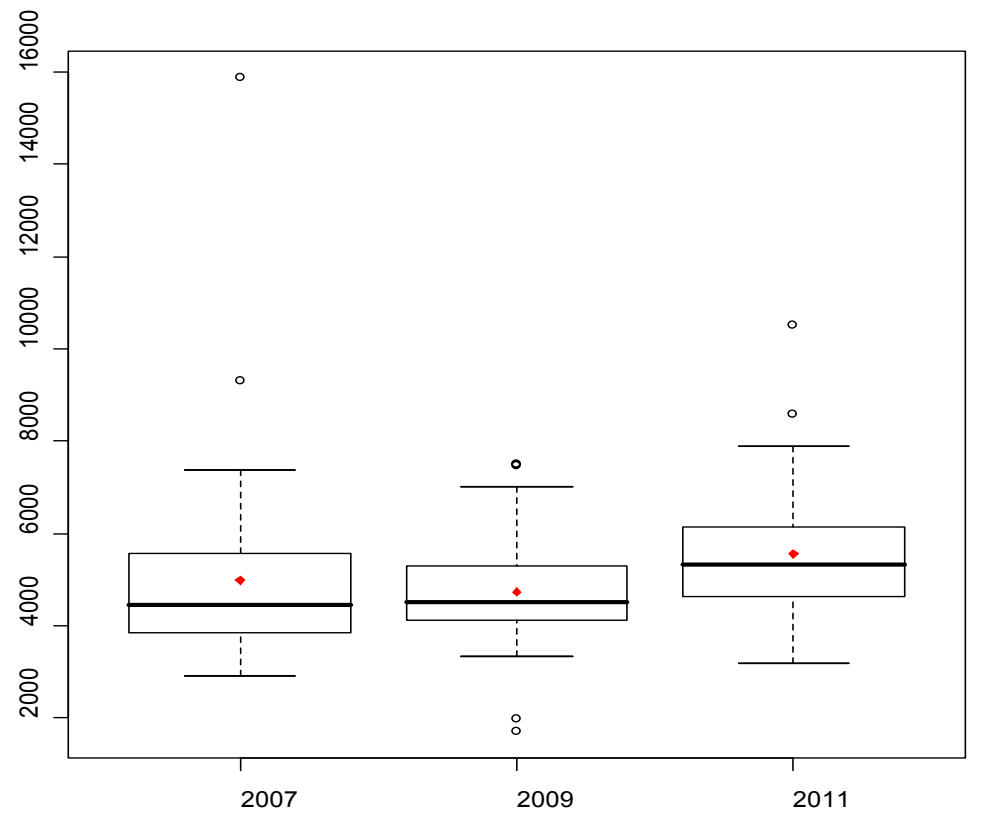

Figura 2. Gasto per capita em educação (2007-2011)

Fonte: Elaborado pelos autores com dados do FNDE (2014) 
Observa-se também que a mediana se manteve abaixo da média nos três períodos. Com relação à amplitude interquartil, em 2007 foi de $R \$ 1.725$ e em 2011 de $R \$ 1.501$. Desta forma, embora a mediana tenha aumentado, a dispersão dos dados se manteve próxima entre os três períodos. Com relação aos outputs, a Tabela 1 apresenta a mediana dos indicadores utilizados no estudo, podendo-se observar que todos indicadores apresentaram melhoras medianas ao longo dos três períodos.

\begin{tabular}{c|c|c|c}
\hline Variável & $\mathbf{2 0 0 7}$ & $\mathbf{2 0 0 9}$ & $\mathbf{2 0 1 1}$ \\
\hline Taxa de Aprovação - Anos Iniciais & 93,9 & 94,6 & 95,6 \\
Taxa de Aprovação - Anos Finais & 90,5 & 90,8 & 91,3 \\
Nota Brasil - Anos Iniciais & 5,43 & 5,75 & 5,86 \\
Nota Brasil - Anos Finais & 4,90 & 5,08 & 5,26 \\
Matrícula - Anos Iniciais & 1567 & 1869 & 1718 \\
Matrícula - Anos Finais & 669 & 695 & 745 \\
\hline
\end{tabular}

Tabela 1. Mediana dos outputs utilizados no modelo.

Fonte: Elaborado pelos autores com dados do INEP (2014).

Já a Figura 3 agrupa o resultado de notas obtidas nos anos iniciais. O gráfico ilustra avanços entre 2007 e 2009, com a mediana em 2009 alinhada ao terceiro quartil de 2007. Contudo, somente entre 2009 e 2011, houve redução da dispersão das notas, com média e mediana próximas às do período anterior. Embora a Figura 2 aponte aumento no gasto per capita, entre 2009 e 2011 observa-se que não houve a conversão direta em melhorias nas notas dos anos iniciais.

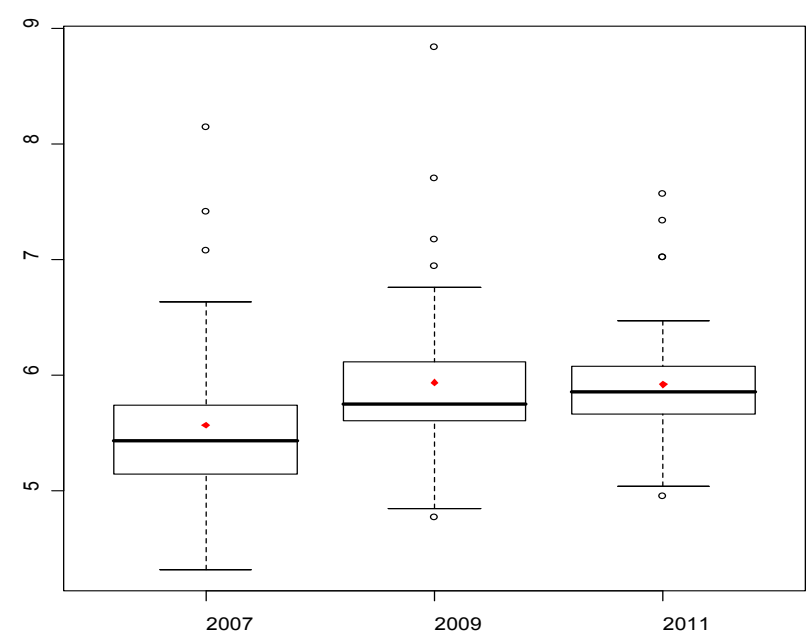

Figura 3. Notas Anos Iniciais (2007-2011).

Fonte: Elaborado pelos autores com os dados INEP (2014).

\subsection{Eficiência Municipal}

A Tabela 2 apresenta um resumo estatístico dos modelos DEA empregados. Conforme modelo CCR-OUT, se todos os municípios tivessem adotado as melhores práticas de alocação e gestão de recursos, seria possível alcançar resultados $23 \%, 29,9 \%$ e $26,7 \%$ melhores, respectivamente para cada ano estudado. Na média global, os resultados poderiam ser $26,5 \%$ melhores. 


\begin{tabular}{|c|c|c|c|c|}
\hline & & 2007 & 2009 & 2011 \\
\hline \multirow{5}{*}{ BCC - INPUT } & Média Geométrica & 0,919 & 0,92 & 0,905 \\
\hline & Média Aritmética & 0,932 & 0,928 & 0,918 \\
\hline & Desvio padrão & 0,133 & 0,118 & 0,139 \\
\hline & Mínimo & 0,408 & 0,579 & 0,533 \\
\hline & Máximo & 1 & 1 & 1 \\
\hline \multirow{5}{*}{ BCC-OUTPUT } & Média Geométrica & 1,012 & 1,01 & 1,01 \\
\hline & Média Aritmética & 1,012 & 1,01 & 1,01 \\
\hline & Desvio padrão & 0,024 & 0,019 & 0,017 \\
\hline & Mínimo & 1 & 1 & 1 \\
\hline & Máximo & 1,109 & 1,081 & 1,068 \\
\hline \multirow{5}{*}{ CCR-INPUT } & Média Geométrica & 0,813 & 0,77 & 0,789 \\
\hline & Média Aritmética & 0,832 & 0,794 & 0,812 \\
\hline & Desvio padrão & 0,169 & 0,193 & 0,178 \\
\hline & Mínimo & 0,408 & 0,446 & 0,331 \\
\hline & Máximo & 1 & 1 & 1 \\
\hline \multirow{5}{*}{ CCR-OUTPUT } & Média Geométrica & 1,23 & 1,299 & 1,267 \\
\hline & Média Aritmética & 1,264 & 1,343 & 1,314 \\
\hline & Desvio padrão & 0,327 & 0,357 & 0,408 \\
\hline & Mínimo & 1 & 1 & 1 \\
\hline & Máximo & 2,452 & 2,241 & 3,017 \\
\hline
\end{tabular}

Tabela 2. Resumo Estatístico - Resultados DEA.

Fonte: Elaborado pelos autores.

O modelo com orientação para output busca os melhores resultados a partir do nível de recursos atuais. No modelo orientado para input, altera-se a óptica de análise: dado o nível de resultados obtidos, verificam-se quais DMUs foram mais eficientes na aplicação de recursos (gastos efetuados). No modelo BCC-INPUT, somente 21 das 47 DMUs estudadas são destaques de benchmarking por atingirem eficiência igual a 1 nos três períodos avaliados (Tabela 3). Ou seja, menos da metade dos munícipios estudados empregou o melhor nível de insumos possível para produzir os resultados educacionais obtidos.

\begin{tabular}{c|c|c|c|c|c|c}
\hline & \multicolumn{2}{|c|}{2007} & \multicolumn{2}{|c|}{2009} & \multicolumn{2}{c}{2011} \\
\hline DMU & Gasto & BCC-INPUT & Gasto & BCC-INPUT & Gasto & BCC-INPUT \\
Cajamar & Alto & 1,00 & Baixo & 1,00 & Alto & 1,00 \\
Dois Córregos & Alto & 1,00 & Baixo & 1,00 & Alto & 1,00 \\
Fernando Prestes & Alto & 1,00 & Alto & 1,00 & Alto & 1,00 \\
Fernandópolis & Alto & 1,00 & Alto & 1,00 & Alto & 1,00 \\
Praia Grande & Alto & 1,00 & Alto & 1,00 & Alto & 1,00 \\
Ribeirão Corrente & Alto & 1,00 & Alto & 1,00 & Baixo & 1,00 \\
Santana de Parnaíba & Alto & 1,00 & Baixo & 1,00 & Alto & 1,00 \\
São José dos Campos & Alto & 1,00 & Alto & 1,00 & Alto & 1,00 \\
Tanabi & Alto & 1,00 & Alto & 1,00 & Alto & 1,00 \\
Adolfo & Baixo & 1,00 & Alto & 1,00 & Baixo & 1,00 \\
Cajuru & Baixo & 1,00 & Baixo & 1,00 & Baixo & 1,00 \\
Capão Bonito & Baixo & 1,00 & Baixo & 1,00 & Baixo & 1,00 \\
Conchal & Baixo & 1,00 & Alto & 1,00 & Alto & 1,00
\end{tabular}




\begin{tabular}{|c|c|c|c|c|c|c|}
\hline Cosmópolis & Baixo & 1,00 & Baixo & 1,00 & Baixo & 1,00 \\
\hline Elisário & Baixo & 1,00 & Alto & 1,00 & Baixo & 1,00 \\
\hline Iperó & Baixo & 1,00 & Baixo & 1,00 & Baixo & 1,00 \\
\hline Lindóia & Baixo & 1,00 & Baixo & 1,00 & Baixo & 1,00 \\
\hline Mairinque & Baixo & 1,00 & Baixo & 1,00 & Baixo & 1,00 \\
\hline Novais & Baixo & 1,00 & Baixo & 1,00 & Baixo & 1,00 \\
\hline Pedra Bela & Baixo & 1,00 & Alto & 1,00 & Baixo & 1,00 \\
\hline Taboão Da Serra & Baixo & 1,00 & Baixo & 1,00 & Alto & 1,00 \\
\hline Novo Horizonte & Alto & 0,70 & Baixo & 1,00 & Baixo & 1,00 \\
\hline Guarujá & Alto & 1,00 & Alto & 0,99 & Alto & 1,00 \\
\hline Catanduva & Baixo & 1,00 & Alto & 0,96 & Baixo & 1,00 \\
\hline Igarapava & Baixo & 1,00 & Baixo & 0,95 & Baixo & 1,00 \\
\hline Urupês & Baixo & 1,00 & Baixo & 0,92 & Baixo & 1,00 \\
\hline Mauá & Alto & 0,41 & Baixo & 0,91 & Baixo & 1,00 \\
\hline Buritizal & Alto & 1,00 & Alto & 0,90 & Baixo & 1,00 \\
\hline Guará & Baixo & 0,91 & Baixo & 0,83 & Baixo & 1,00 \\
\hline Jaú & Baixo & 1,00 & Baixo & 0,75 & Baixo & 1,00 \\
\hline Águas De Lindóia & Alto & 0,54 & Alto & 0,67 & Alto & 1,00 \\
\hline Uchoa & Baixo & 0,96 & Alto & 1,00 & Baixo & 0,93 \\
\hline Martinópolis & Baixo & 0,86 & Baixo & 1,00 & Alto & 0,91 \\
\hline Guaratinguetá & Alto & 0,68 & Alto & 0,85 & Alto & 0,90 \\
\hline Pradópolis & Alto & 0,91 & Baixo & 0,76 & Baixo & 0,90 \\
\hline Charqueada & Baixo & 1,00 & Baixo & 0,75 & Baixo & 0,86 \\
\hline Monte Alto & Alto & 1,00 & Baixo & 0,58 & Baixo & 0,84 \\
\hline Caraguatatuba & Alto & 0,76 & Alto & 0,86 & Alto & 0,83 \\
\hline Ubarana & Baixo & 0,87 & Baixo & 1,00 & Baixo & 0,81 \\
\hline Jeriquara & Alto & 1,00 & Alto & 1,00 & Alto & 0,78 \\
\hline Botucatu & Alto & 0,85 & Alto & 0,78 & Alto & 0,76 \\
\hline Viradouro & Baixo & 0,87 & Alto & 1,00 & Alto & 0,71 \\
\hline São Carlos & Alto & 0,97 & Alto & 0,86 & Alto & 0,69 \\
\hline Itirapina & Baixo & 1,00 & Baixo & 0,60 & Alto & 0,59 \\
\hline José Bonifácio & Baixo & 0,79 & Baixo & 1,00 & Alto & 0,56 \\
\hline Cubatão & Alto & 1,00 & Alto & 1,00 & Alto & 0,55 \\
\hline Araraquara & Alto & 0,72 & Alto & 0,70 & Alto & 0,53 \\
\hline
\end{tabular}

Tabela 3. Gasto municipal e eficiência.

Fonte: Elaborado pelos autores.

Destacam-se neste modelo as DMUs dos municípios paulistas Adolfo, Elisário, Novaes e Pedra Bela. Nos três períodos analisados, estes municípios alcançaram índice de eficiência igual a 1. Dado o nível de gasto do grupo estudado, eles podem ser considerados benchmarking na obtenção de resultados educacionais.

Conforme média geométrica do modelo BCC-INPUT, que consta na Tabela 2, as DMUs ao todo apresentam eficiência de aproximadamente 92\% para 2007 e 2009 e de $91 \%$ para 2011. Numa situação hipotética, em que todos os municípios estivessem na fronteira de eficiência, 
seria possível obter os mesmos outputs com menos $8 \%$ do gasto municipal em 2007 e 2009 e $9 \%$ no ano de 2011. Ressalta-se que os valores resultantes da ineficiência técnica da gestão poderiam ser convertidos na expansão/melhoria da rede de ensino de maneira mais eficiente. Blackburn, Brennan e Ruggiero (2014) ao avaliarem escolas da Austrália encontram também nível extra de gastos (20\%), contudo, maiores que os níveis verificados neste estudo.

A Tabela 4 apresenta, em valores absolutos, a economia possível de recursos referentes ao três períodos analisados (dados atualizados para jun/2014).

\begin{tabular}{c|cr|rr|rr}
\hline Economia Possível no Ano & \multicolumn{2}{|c|}{ Despesas de Pessoal } & \multicolumn{2}{c|}{ Outras despesas } & \multicolumn{2}{c}{ Investimentos } \\
\hline 2007 & $\mathrm{R} \$$ & $59.196 .225,65$ & $\mathrm{R} \$$ & $31.635 .200,38$ & $\mathrm{R} \$$ & $12.846 .008,55$ \\
2009 & $\mathrm{R} \$$ & $64.753 .665,85$ & $\mathrm{R} \$$ & $35.613 .302,81$ & $\mathrm{R} \$$ & $6.886 .365,24$ \\
2011 & $\mathrm{R} \$$ & $87.909 .443,27$ & $\mathrm{R} \$$ & $52.745 .469,19$ & $\mathrm{R} \$$ & $12.292 .427,25$ \\
Total & $\mathrm{R} \$$ & $\mathbf{2 1 1 . 8 5 9 . 3 3 4 , 7 8}$ & $\mathrm{R} \$$ & $\mathbf{1 1 9 . 9 9 3 . 9 7 2 , 3 8}$ & $\mathrm{R} \$ \mathbf{3 2 . 0 2 4 . 8 0 1 , 0 5}$ \\
\hline
\end{tabular}

Tabela 4. Economia de recursos possível segundo modelo BCC-INPUT.

Fonte: Elaborado pelos autores.

Parte desta ineficiência pode estar relacionada ao porte inadequado, gerando uma ineficiência de escala, medida usualmente pela diferença entre BCC-INPUT e CCR-INPUT, a ineficiência de escala responde respectivamente por 10,6\%, 15,00\% e 11,6\% em relação ao total da ineficiência observada.

Para lidar com a questão da estrutura de ensino (escala), Rosano-Peña, Albuquerque e Marcio (2012) sugerem que redes municipais superdimensionadas podem se descentralizar criando unidades escolares nos municípios que operam em ótima escala. Já as subdimensionadas, poderiam utilizar sistemas de consórcios ou sistemas intermunicipais de ensino, favorecendo o ganho em escala. Ressalta-se que dos 47 municípios analisados no estudo, novamente, os municípios de Adolfo, Elisário, Novaes e Pedra Bela são destaque, pois mantiveram eficiência em escala durante os três períodos.

\subsection{Níveis de Gastos e Eficiência}

Com intuito de avaliar o nível de gasto governamental e a eficiência dos municípios, as DMUs foram divididas em dois grupos utilizando a mediana do gasto per capita para cada ano. Municípios com gasto acima da mediana foram classificados como "Alto" e municípios com gasto abaixo como "Baixo".

Os resultados para cada ano também constam na Tabela 3. As linhas sombreadas marcam os 21 municípios que se mantiveram na fronteira de eficiência nos três anos estudados, dentre eles há municípios classificados com gastos altos e baixos. Os demais municípios ficaram na fronteira de eficiência em pelo menos um dos períodos, exceto Araraquara que se apresentou ineficiente nos três anos analisados.

A Figura 4 ilustra a eficiência média dos grupos. Os municípios com baixo gasto tem eficiência média superior em dois dos três períodos estudados. Já em 2009 ambos os grupos se aproximam da eficiência média geral. Contudo, não foi possível verificar diferenças estatisticamente significativas de médias entre os grupos com gasto, alto ou baixo. Os resultados corroboram com o trabalho de Marlow (2000) que constatou não existir uma relação entre recursos educacionais e desempenho dos alunos. A fronteira de eficiência encontrada no estudo foi composta por municípios tanto com gastos acima, quanto abaixo da mediana per capita. Desta forma, existem municípios com gasto baixo, mas com alta eficiência na aplicação destes recursos. Assim, embora Sobreira e Campos (2008) tenham encontrado relação positiva entre gastos e desempenho escolar, utilizando painel de dados, quando avaliado o nível de eficiência, não foi possível corroborar tal resultado. 


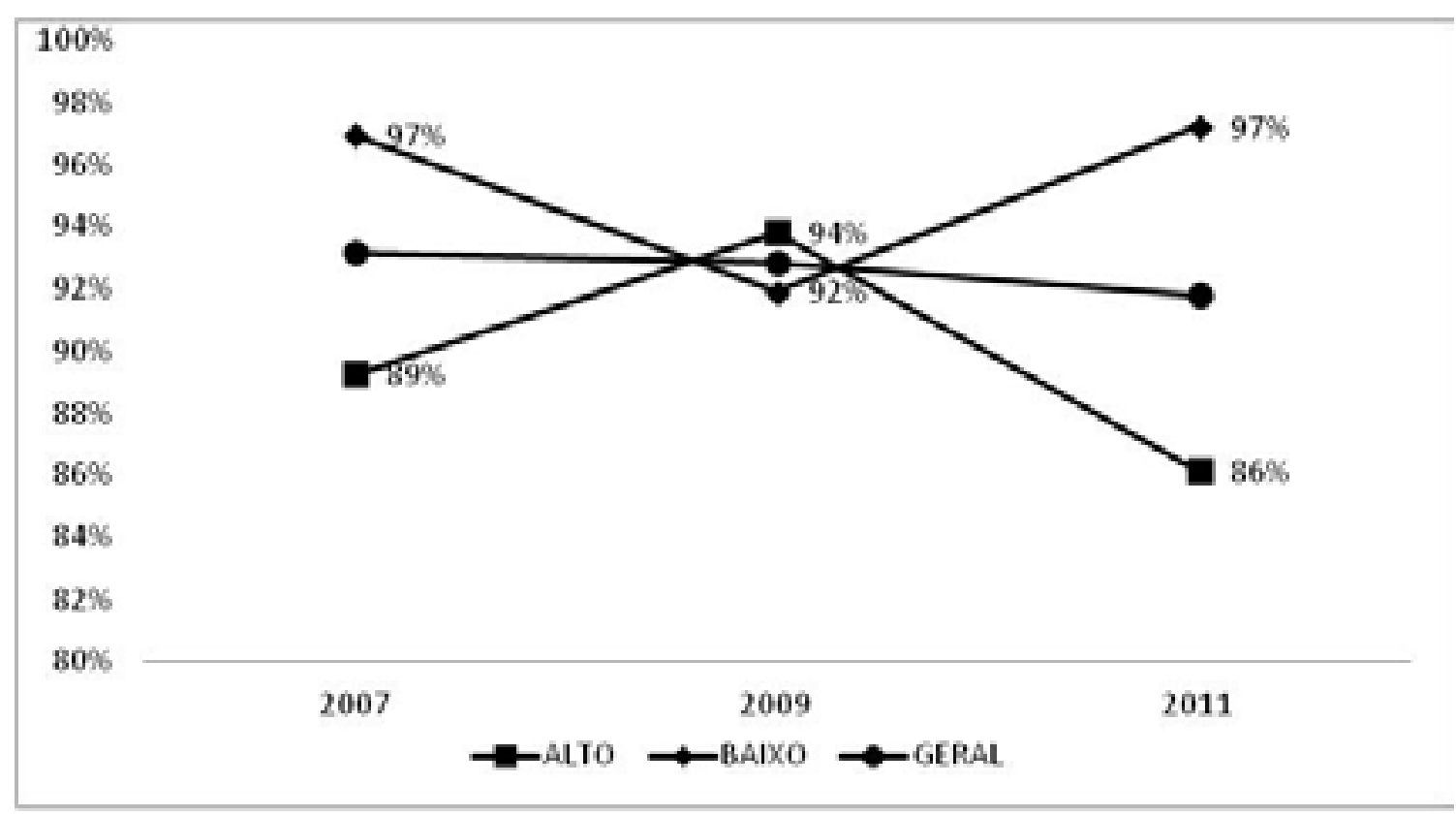

Figura 4. Eficiência Média (BCC-INPUT) em função do Gasto per capita.

Fonte: Elaborado pelos autores.

Também foi analisada a ineficiência em escala dos grupos. A Tabela 5 apresenta o quanto da ineficiência total pode ser originário de uma ineficiência em escala, os resultados obtidos quando considerado o gasto per capita aparentam ser mais condizentes do que a média geral $(10,6 \%, 15,00 \%$ e $11,6 \%)$ anteriormente encontrada. Grupos com gasto alto apresentaram maior ineficiência de escala em todos os períodos. Adicionalmente, a aplicação do teste Wilcoxon (com intervalo de confiança de $95 \%$ ) retornou que municípios com gasto abaixo da mediana têm menor ineficiência, em escala, que municípios com gasto acima da mediana.

\begin{tabular}{c|c|c|c}
\hline Relação & $\mathbf{2 0 0 7}$ & $\mathbf{2 0 0 9}$ & $\mathbf{2 0 1 1}$ \\
\hline Alto & $18 \%$ & $20 \%$ & $19 \%$ \\
Baixo & $3 \%$ & $7 \%$ & $5 \%$ \\
\hline
\end{tabular}

Tabela 5. Ineficiência média de escala em relação ao gasto per capita.

Fonte: Elaborado pelos autores.

Estes resultados podem indicar que o aumento do gasto em educação, quando não planejado adequadamente, gera unidades superdimensionadas, impactando na eficiência geral da unidade tomadora de decisão. Desta forma, a unidade apresenta gestão eficiente para o nível possível de resultados alcançados, mas tem capacidade produtiva excessiva em virtude do gasto per capita mais alto, apresentando piores resultados em eficiência técnica de escala.

\section{CONSIDERAÇÕES FINAIS}

Este estudo teve por objetivo verificar e compreender a eficiência de uma amostra de municípios paulistas na aplicação de recursos em educação básica nos anos de 2007, 2009 e 2011. Para tanto, foi utilizada a Análise Envoltória de Dados (DEA), com os inputs referentes aos recursos financeiros empregados no ensino fundamental e os outputs referentes ao desempenho dos alunos no respectivo nível de ensino. 
Os resultados indicaram aumento do gasto total (inputs) em educação entre os períodos analisados e, de 2007 para 2011 registrou-se um crescimento real de 31\% em todos os municípios do grupo.

Quanto aos outputs, observou-se também uma melhoria nos indicadores utilizados nos três períodos. Entretanto, embora haja um aumento do gasto per capita entre 2009 e 2011 observa-se que não houve a conversão direta em melhorias nas notas dos alunos. Conforme modelo orientado para output, se todos os municípios estudados tivessem adotado as melhores práticas de alocação e gestão de recursos, seria possível alcançar resultados, em média, 26,5\% melhores.

Já em relação ao nível de gasto municipal, não houve diferença significativa de média entre os grupos de municípios com gastos alto e baixo. Desta maneira , não é possível afirmar se municípios que gastam acima ou abaixo da mediana tem maior eficiência técnica na geração de resultados educacionais.

Por outro lado, os grupos com gasto alto apresentaram uma maior ineficiência de escala em todos os períodos. Estes resultados corroboram com os estudos de Rosano-Peña, Albuquerque e Marcio (2012) que concluem que a maior alocação de recursos não proporciona melhores resultados se estes forem aplicados de maneira ineficiente.

Desta forma, a unidade apresentou gestão eficiente para o nível possível de resultados alcançados, mas tem capacidade produtiva excessiva em virtude do gasto per capita mais alto. Assim, estas unidades superdimensionadas acabaram por afetar a eficiência geral da unidade tomadora de decisão (DMU).

Os municípios de Adolfo, Elisário, Novaes e Pedra Bela, são de pequeno porte, com gasto baixo, exceto em 2009, e apresentaram eficiência em todos os modelos analisados. Sugere-se como pesquisa futura o entendimento mais aprofundado das melhores práticas adotadas por estas DMUs, como meio de benchmarking para redes educacionais. O único município que se apresentou como ineficiente nos três períodos analisados foi Araraquara. Sugere-se avaliar o impacto do porte municipal e suas especificidades para melhorar a eficiência deste município no alcance de resultados educacionais favoráveis.

Com relação às contribuições acadêmicas e gerencias, é possível destacar, sobretudo, a importância de uma ação coordenada entre formação e prática dos gestores escolares. Tais contribuições estão baseadas em dois resultados relevantes que a pesquisa empreendida acrescentou ao debate: (i) encontrar relação positiva entre gastos e desempenho escolar não é condição suficiente para confirmar nível de eficiência elevado e (ii) a avaliação do ensino fundamental pelas métricas de eficiência de escala apontaram para estratégias efetivas na redução de custos que pondere o porte ideal das unidades.

Mostra-se crucial, neste sentido, promover uma formação orientada ao gerenciamento eficiente de recursos, incentivando a qualificação e a formação continuada de gestores, assim como a promoção de debates sobre programas e propostas pedagógicas e gerenciais. Além disso, a necessidade de inclusão na grade curricular dos cursos de licenciatura de disciplinas de gestão pública, que abordem conceitos de receitas, custos, despesas, estoques, entre outros aspectos importantes para uma gestão eficiente da escola de educação básica. Também se recomenda a implantação do gerenciamento por meio de indicadores que subsidiem à tomada de decisões e permita uma melhor alocação dos recursos.

Ademais, este estudo apresentou limitações quanto ao número de DMUs analisadas, visto que para os três anos só havia dados disponíveis de 47 municípios dos 645 do Estado de São Paulo. Além disso, como limitação, o método e a amostra não permitem generalizações para a população em estudo. Sugere-se, portanto como pesquisa futura a aplicação de métodos paramétricos como fronteira estocástica, por exemplo. Adicionalmente, estudos futuros também podem avaliar o impacto na eficiência de variáveis externas não controláveis pelas DMUs como características socioeconômicas e habilidades conceituais prévias. 


\section{REFERÊNCIAS}

ALBERNAZ, Ângela; FERREIRA, Francisco de Hollanda Guimarães; FRANCO, Creso. Qualidade e Equidade no Ensino Fundamental Brasileiro. Pesquisa e Planejamento Econômico, v. 32, n. 03, p. 453-476, 2002.

AMBROZINI, Marcelo Augusto; NAGANO, Marcelo Seido; MERLO, Edgard Monforte. O Desempenho do Setor de Varejo no Brasil e suas Mudanças no Período Recente. Anais do VI SEMEAD, v. 6, p. 1-12, 2003.

BANKER, Rajiv D.; CHARNES, Abraham; COOPER, William W. Some Models for Estimating Technical and Scale Inefficiencies in Data Envelopment Analysis. Management Science, v. 30, p. 1078 - 1092, 1984.

BARROS, Ricardo Paes; MENDONÇA, Rosane; SANTOS, Daniel Domingues; QUINTAES, Giovani. Determinantes do Desempenho Educacional no Brasil. Pesquisa e Planejamento Econômico, v. 31, n.01, p. 1-42, 2001.

BIFULCO, Robert; BRETSCHNEIDER, Stuart. Estimating school efficiency: a comparison of methods using simulated data. Economics of Education Review, v. 20, p. 417-429, 2001.

BLACKBURN, Vincent; BRENNAN, Shae; RUGGIERO, John. Measuring efficiency in Australian Schools: A preliminary analysis. Socio-Economic Planning Sciences, v. 48, n. 1, p. 4-9, mar. 2014.

BOGETOFT, Peter. OTTO, Lars. Benchmarking with DEA, SFA, and R, v. 157, 2011.

BONAMINO, Alicia Maria Catalano; ALVES, Fátima; CAZELLI, Sibele; FRANCO, Creso. Os efeitos das diferentes formas de capital no desempenho escolar: um estudo à luz de Bourdieu e de Coleman. Revista Brasileira de Educação, v. 15, p. 487-499, 2010.

BRASIL. Lei no 12.527, de 18 de Novembro de 2011. 2011. Disponível em: < http://www.planalto. gov.br/ccivil_03/_ato2011-2014/2011/lei/l12527.htm > Acesso em: 18 jun. 2014.

CHARNES, Abraham; COOPER, William W.; RHODES, Edwardo L. Measuring the efficiency of decision making units. European journal of operational, v. 2, p. 429-444, 1978.

CONROY, Stephen J.; ARGUEA, Nestor M. An estimation of technical efficiency for Florida public elementary schools. Economics of Education Review, v. 27, n. 6, p. 655-663, 2008.

DELORS, Jacques.; AL-MUFTI, In'am.; AMAGI, Isao.; et al. Educação: um tesouro a descobrir. Relatório para a UNESCO da Comissão Internacional sobre Educação para o Séc. XXI. São Paulo: Cortez Editora, 1998.

DIDONET, Simone Regina; VILLAVICENCIO, Guillermo Javier Díaz. Orientácion al mercado y eficiencia em los supermercados: evidencias empíricas. Revista de Administração da UFSM, v. 1, n. 1, p. 134-152, 2008.

FARRELL, Michael James.The measurement of productive efficiency. Journal of the Royal Statistical Society. Series A, v. CXX, n. part 3, p. 253-290, 1957.

FARIA, Flávia Peixoto; JANNUZZI, Paulode Martino e SILVA, Silvano José. Da. Eficiência dos gastos municipais em saúde e educação : uma investigação através da análise envoltória no estado do Rio de Janeiro. Revista de Administração Pública, v. 42, n. 1, p. 155-177, 2008. 
FNDE, FUNDO NACIONAL DE DESENVOLVIMENTO DA EDUCAÇÃO. Relatórios Municipais. Sistema de Informações sobre Orçamentos Públicos em Educação. 2014. Disponível em: < https://www. fnde.gov.br/siope/relatoriosMunicipais.jsp> Acesso em: 18 jan. 2014

HARRISON, Julie; ROUSE, Paul. Competition and public high school performance. Socio-Economic Planning Sciences, v. 48, n. 1, p. 10-19, mar. 2014.

IBGE. INSTITUTO BRASILEIRO DE GEOGRÁFIA E ESTATístICA. Cidades. 2014. Disponível em: <http://www.cidades.ibge.gov.br/xtras/uf.php?lang=\&coduf=35\&search=sao-paulo> Acesso em: 18 de jan. de 2014

INEP. Instituto Nacional de Estudos e Pesquisas Educacionais Anísio Teixeira. Nota Técnica (2014). 2014. Disponível em: <http://download.inep.gov.br/educacao_basica/portal_ideb/o_que_e_o_ ideb/Nota_Tecnica_n1_concepcaolDEB.pdf>. Acesso em 11 de jan. de 2014.

KIM, Hong-Kyum. Is there a crowding-out effect between school expenditure and mother's child care time? Economics of Education Review, v. 20, n. 1, p. 71-80, Feb. 2001.

MARLOW, Michael. Spending, school structure, and public education quality: evidence from California. Economics of Education Review, v. 19, n. 1, p. 89-106, Feb. 2000.

PORTAL ACTION. Teste binomial exata. Disponível em: <http://www.portalaction.com.br/ content/36-teste-binomial-exata>. Acesso em: 29/01/2013.

RAMOS, M.; SCHABBACH, L. O estado da arte da avaliação de políticas públicas: conceituação e exemplos de avaliação no Brasil. Revista de Administração Pública, v. 46, n. 5, p. 1271-1294, 2012.

RIBEIRO, Patrícia Tavares. A descentralização da ação governamental no Brasil dos anos noventa: desafios do ambiente político-institucional. Ciência \& Saúde Coletiva, v. 14, n. 3, p. 819-828, 2009.

RIBEIRO FILHO, José Francisco; LOPES, Jorge Expedito de Gusmão; PEDERNEIRAS, Marcleide Maria Macedo; SILVA, Alan José Moura; MULATINHO, Caio Eduardo Silva. Recomendações em auditoria operacional: uma prospecção de fragilidades, com base na inteligência competitiva. Revista de Administração da UFSM, v. 3, n. 2, p. 191-204, 2010.

ROSANO-PEÑA, Carlos; AlBuQUerque, Pedro Henrique Melo; MARCIO, Carvalho Jose. A eficiência dos gastos públicos em educação: evidências georreferenciadas nos municípios goianos. Economia Aplicada, v. 16, n. 3, p. 421-443, 2012.

SAMPAIO, Breno; GUIMARÃES, Juliana. Diferenças de eficiência entre ensino público e privado no Brasil. Economia Aplicada, v. 13, n. 1, p. 45-68, 2009.

SAURIN, Valter; LOPES, Ana Lúcia Miranda; COSTA JUNIOR, Newton Carneiro Affonso; GONÇALVES, Carlos Alberto. Medidas de eficiência e retorno de investimento: um estudo nas distribuidoras de energia elétrica brasileiras com base em Data Envelopment Analysis, Índice de Malmquist e ROI. Revista de Administração da UFSM, v. 6, n. 1, p. 25-38, 2013.

SEADE, FUNDAÇÃO SISTEMA ESTADUAIS DE ANÁLISE DE DADOS. Banco de dados educação municipal. 2014. Disponível em: <http://www.seade.gov.br/banco-de-dados/> Acesso: 10 maio 2014 
SILVA, Maurício Corrêa; SOUZA, Fábia Jaiany Viana de; ARAÚJO, Aneide Oliveira. Análise da eficiência dos gastos públicos com educação nas capitais brasileiras. Contexto, v. 13, n. 24, p. 7-21, 2013.

SOBREIRA, Rogério; CAMPOS, Bruno Cesar. Investimento público em educação fundamental e a qualidade do ensino: uma avaliação regional dos resultados do Fundef. Revista de Administração Pública, v. 42, n. 2, p. 327-346., 2008

UNNEVER, James D.; KERCKHOFF, Allan C.; ROBINSON, Timothy J. District variations in the educational resources and student outcomes. Economics of Education Review, v. 19, n. 3, p. 245259, Jun. 2000.

VILLAVICENCIO, Guillermo Javier Díaz; DIDONET, Simone Regina. Eco-eficiencia em la gestión de residuos municipales em Catalunya. Revista de Administração da UFSM, v. 1, n. 2, p. 193-208, 2008.

WALDO, Staffan. On the use of student data in efficiency analysis-Technical efficiency in Swedish upper secondary school. Economics of Education Review, v. 26, p. 173-185, 2007.

WILBERT, Marcelo Driemeyer; D’ABREU, Erich Cesar Cysne Frota. Eficiência dos Gastos Públicos na Educação: Análise dos Municípios do Estado de Alagoas. Advances in Specific and Applied Accounting, v. 6, n. 3, p. 348-372, 2013.

WOESSMANN, Ludger. Why students in some countries do better? International evidence on the importance of education policy. Education Matters, v.1, n.2, p. 67-74, 2003.

ZOGHBI, Ana Carolina Pereira; MATOS, Enlinson Henrique Carvalho de; ROCHA, Fabiana Fontes; ARVATE, Paulo Roberto. Mensurando o Desempenho e a Eficiência dos Gastos Estaduais em Educação Fundamental e Média. Estudos Econômicos, v. 39, n. 4, p. 785-809, 2009. 